\title{
Ambulatory arterial stiffness index predicts stroke in a general population
}

\author{
Tine W. Hansen ${ }^{\mathrm{a}, \mathrm{b}, \mathrm{c}}$, Jan A. Staessen ${ }^{\mathrm{d}}$, Christian Torp-Pedersen ${ }^{\mathrm{b}}$, Susanne \\ Rasmussen ${ }^{\mathrm{c}}$, Yan Li ${ }^{\mathrm{e}}$, Eamon Dolan ${ }^{\mathrm{f}}$, Lutgarde Thijs ${ }^{\mathrm{d}}$, Ji-Guang Wang ${ }^{\mathrm{e}}$, \\ Eoin $\mathrm{O}^{\prime}$ Brien $^{\dagger}, \mathrm{Hans}_{\mathrm{Ibsen}}{ }^{\mathrm{c}}$ and Jørgen Jeppesen ${ }^{\mathrm{c}}$
}

Objective The ambulatory arterial stiffness index (AASI) can be computed from individual 24-h blood pressure recordings.

Methods We investigated the prognostic value of AASI and 24-h pulse pressure in a random sample of 1829 Danes, aged 40-70 years. We adjusted for sex, age, body mass index, mean arterial pressure, smoking, diabetes, ratio of total to high-density lipoprotein cholesterol, and history of cardiovascular disease with Cox regression.

Results Over a median follow-up of 9.4 years, incidences of fatal and nonfatal endpoints were $\mathbf{4 0}$ for stroke, 150 for coronary heart disease, and $\mathbf{2 1 2}$ for cardiovascular events. In fully adjusted models, the hazard ratios associated with $1 \mathrm{SD}$ increase $(0.14 \mathrm{U})$ in the AASI were $1.62(95 \%$ confidence interval, 1.14-2.28; $P=0.007$ ) for stroke, $0.96(0.80-1.14 ; P=0.62)$ for coronary heart disease, and $1.06(0.91-1.23 ; P=0.49)$ for cardiovascular events. None of these ratios reached significance for pulse pressure $(P>0.47)$. The AASI still predicted stroke after excluding individuals with previous cardiovascular disease or after adjustment for systolic and/or diastolic blood pressure instead of mean arterial pressure.

Conclusions In a randomly recruited European population, the AASI was a strong predictor of stroke, beyond traditional

\section{Introduction}

Stiffness of the central arteries, as reflected by the aortic pulse wave velocity [1-5], predicts the incidence of cardiovascular complications over and beyond traditional risk factors and 24-h mean arterial pressure. Most methods to measure arterial stiffness require special equipment and trained observers. In contrast, the ambulatory arterial stiffness index (AASI), defined as one minus the regression slope of diastolic on systolic blood pressure in individuals, can be determined from regular 24-h ambulatory blood pressure recordings [6,7]. The AASI shows high correlations with several measures of arterial stiffness, including the aortic pulse wave velocity, the central and peripheral pulse pressures, and the systolic augmentation index [6]. In the Dublin Outcome Study, both the AASI and the 24-h pulse pressure, while adjusting for other risk factors, predicted cardiovascular mortality. Furthermore, compared with the 24-h pulse pressure, the AASI was a stronger predictor of fatal stroke, cardiovascular risk factors, including the mean arterial pressure and pulse pressure. J Hypertens 24:2247-2253 (C) 2006 Lippincott Williams \& Wilkins.

Journal of Hypertension 2006, 24:2247-2253

Keywords: arterial stiffness, pulse pressure, cardiovascular disease, risk factors, epidemiology

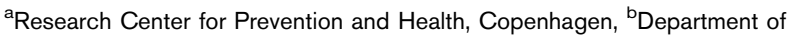
Cardiology, Bispebjerg University Hospital, Copenhagen, ${ }^{\circ}$ Medical Department M, Glostrup University Hospital, Copenhagen, Denmark, d'Studies Coordinating Centre, Division of Hypertension and Cardiovascular Rehabilitation, Department of Cardiovascular Diseases, University of Leuven, Belgium, ${ }^{\mathrm{e}}$ Centre for Epidemiological Studies and Clinical Trials, Ruijin Hospital, Shanghai Institute of Hypertension, Shanghai Second Medical University, Shanghai, China and ${ }^{f}$ ADAPT Centre, Beaumont Hospital, and Department of Clinical Pharmacology, Royal College of Surgeons in Ireland, Dublin, Ireland

Correspondence and requests for reprints to Tine Willum Hansen, MD, PhD, Bispebjerg Hosptal, Y-forskning bygning 40, Bispebjerg Bakke, DK-2400 Copenhagen NV, Denmark

Tel: +45 35316161 ; fax: +45 397518 08; e-mail: tw@heart.dk

Sponsorship: The Danish Heart Foundation (grant 01-2-9-9A-22914), the Danish Medical Association Research Fund/Volten, and the Danish Pharmaceutical Association provided grants in support of the present research. Research at the Studies Coordinating Centre in Leuven, Belgium is supported by grants from the Fonds voor Wetenschappelijk Onderzoek Vlaanderen (Brussels; G.0453.05) and the University of Leuven (Onderzoekstoelage OT/05/49). The Bilateral Scientific and Technological Cooperation between China and Flanders (grant BIL02/10, Ministry of the Flemish Community, Brussels, Belgium) supported the postdoctoral research of Y.L. in Leuven and travel of J.A.S. to Shanghai.

Received 18 March 2006 Accepted 28 June 2006

especially in normotensive individuals, whereas the opposite was true for the 24-h pulse pressure in relation to cardiac mortality [7].

Ambulatory blood pressure monitoring is used more and more in clinical practice for the diagnosis and management of hypertension [8]. While the AASI might refine the risk stratification based on ambulatory blood pressure measurement, its use cannot be recommended before the Irish outcome results [7] are replicated in other cohorts. We therefore investigated to what extent the AASI predicted fatal and nonfatal cardiovascular complications in middle-aged and older individuals randomly recruited from the population of Copenhagen.

\section{Methods}

Study population

The Ethics Committee of Copenhagen County approved the Monitoring of Trends and Determinants in 
Cardiovascular Disease health survey [9]. The study was conducted in accordance with the Helsinki Declaration [10]. Participants provided informed written consent. In 1982-1984, we randomly selected residents from Copenhagen County with the goal of recruiting an equal number of women and men aged 30, 40, 50, and 60 years [11]. At baseline, the participation rate was $82.6 \%$. In 1993-1994, the 3785 former participants were invited for a follow-up examination at the Research Center for Prevention and Health in Glostrup, of whom $2656(70.2 \%)$ renewed informed written consent and were examined [11]. For the present analysis, we excluded 827 individuals because they were unwilling to have their ambulatory blood pressure measured (304 women and 270 men), because they had fewer than 14 daytime or seven night-time blood pressure readings (163 women and 77 men) [12], or because their ambulatory blood pressure had been recorded during nighttime shifts (three women and 10 men). The number of participants statistically analyzed therefore totaled 1829 (68.9\% of those with a follow-up examination).

\section{Data collection}

At the research center, a trained nurse measured anthropometric characteristic. The body mass index was weight in kilograms divided by height in meters squared. We programmed validated [13] Takeda TM-2421 recorders (A\&D, Tokyo, Japan) to obtain blood pressure recordings at intervals of $15 \mathrm{~min}$ from 0700 to $2300 \mathrm{~h}$, and every $30 \mathrm{~min}$ from 2300 to $0700 \mathrm{~h}$. For analysis we only used the oscillometric measurements. We computed the withinsubject 24-h means of the ambulatory measurements with weights according to the time interval between successive readings. Hypertension was defined as an awake ambulatory blood pressure of at least $135 \mathrm{mmHg}$ systolic or $85 \mathrm{mmHg}$ diastolic [8], or as the use of antihypertensive drugs.

From unedited 24-h recordings, we computed for each participant the regression slope of diastolic on systolic blood pressure $[6,7]$. We did not force the regression line through the origin (intercept $=0$ ) because, during diastole when flow drops to zero, such a phenomenon does not occur for blood pressure [14]. We defined the AASI as one minus the regression slope. The stiffer the arterial tree, the closer the regression slope and AASI are to zero and unity, respectively [6,7]. In addition, based on individual recordings, we computed the pulse pressure as the difference between the 24-h systolic and diastolic blood pressures and mean arterial pressure as the 24-h diastolic blood pressure plus one-third of the pulse pressure.

Venous blood samples, collected after overnight fasting, were analyzed by standard automated methods for lipids and blood glucose. According to published criteria [15], diabetes mellitus was defined as a fasting blood glucose level of at least $7.0 \mathrm{mmol} / \mathrm{l}$ or as the use of antidiabetic drugs. The participants completed a self-administrated questionnaire inquiring into their past and current medical history, intake of medications, and lifestyle.

\section{Ascertainment of events}

We obtained vital status via the Danish Civil Registration System, cause of death from blinded adjudication of the diseases on the death certificates, and nonfatal events from the Danish National Health Register [16]. The endpoints considered in the present analysis were fatal and nonfatal stroke (ICD-8 codes 431, 433, or 434 or ICD-10 codes I61 or I63), fatal and nonfatal coronary heart disease (ICD-8 code 410-414 or ICD-10 codes I20-I25), and a composite endpoint consisting of cardiovascular mortality, coronary heart disease, and stroke.

\section{Statistical analysis}

For statistical analysis, we used SAS software (version 9.1; SAS Institute, Cary, North Carolina, USA). To compare means, we used the standard normal z-test for large samples or analysis of variance, and for proportions the chi-squared statistic. In the outcome analysis, for participants who experienced multiple events, we only considered the first. We calculated relative hazard ratios by multiple Cox regression with adjustment for baseline characteristics including sex, age, body mass index, mean arterial pressure, smoking, diabetes mellitus, the ratio of total to high-density lipoprotein (HDL) serum cholesterol, and a history of cardiovascular disease. To test for heterogeneity in the relations of outcome with the AASI, we forced appropriate interaction terms into the regression models. In further analyses, we dichotomized the AASI and pulse pressure, using the upper boundary of the 95th prediction interval for individual data points in relation to age in the Belgian, Chinese, and Irish normotensive individuals enrolled in the International Database on Ambulatory Blood Pressure Monitoring [17]. Cut-off limits by decade of age ranged from 0.62 at 40 years to 0.71 at 70 years for AASI and from $57 \mathrm{mmHg}$ at 40 years to $59 \mathrm{mmHg}$ at 70 years for the $24-\mathrm{h}$ pulse pressure $[6,7]$. We modeled the probability of the 10 -year incidence of stroke at different levels of mean arterial pressure and pulse pressure, using Cox regression while controlling for sex and age. Statistical significance was a $P$ value of 0.05 or less on two-sided tests.

\section{Results}

\section{Baseline characteristics of the participants}

The 1829 participants included 858 (46.9\%) women and $796(43.5 \%)$ patients with ambulatory hypertension, of whom $186(23.4 \%)$ were taking antihypertensive drugs. Mean $\pm \mathrm{SD}$ values were $55.5 \pm 10.7$ years for age, $0.56 \pm 0.14 \mathrm{U}$ for the AASI, and $52.3 \pm 9.0 \mathrm{mmHg}$ for pulse pressure. Table 1 presents the characteristics of the study population across quintiles of the AASI distribution. Between-quintile differences were significant $(P<0.03)$ except for the prevalence of smoking and 
Table 1 Clinical characteristics across quintiles of the ambulatory arterial stiffness index (AASI)

\begin{tabular}{|c|c|c|c|c|c|c|}
\hline & \multicolumn{5}{|c|}{ AASI percentile limits } & \multirow[b]{2}{*}{$P$ value } \\
\hline & $\leq 0.43$ & $>0.43$ to $\leq 0.52$ & $>0.52$ to $\leq 0.60$ & $>0.60$ to $\leq 0.68$ & $>0.68$ & \\
\hline Number of individuals & 366 & 366 & 366 & 366 & 365 & \\
\hline Women (\%) & 36.3 & 44.3 & 45.9 & 50.8 & 57.3 & $<0.001$ \\
\hline Age (years) & $51.8 \pm 10.0$ & $56.3 \pm 10.5$ & $56.8 \pm 10.9$ & $56.9 \pm 10.6$ & $55.6 \pm 10.8$ & $<0.001$ \\
\hline Body mass index $\left(\mathrm{kg} / \mathrm{m}^{2}\right)$ & $25.9 \pm 3.8$ & $26.2 \pm 4.0$ & $26.1 \pm 4.0$ & $25.9 \pm 4.2$ & $25.7 \pm 4.2$ & $<0.001$ \\
\hline \multicolumn{7}{|l|}{ Risk factors } \\
\hline Smoking (\%) & 45.6 & 43.2 & 47.0 & 46.2 & 38.6 & 0.15 \\
\hline Hypertension (\%) ${ }^{\mathrm{a}}$ & 47.8 & 46.7 & 47.0 & 41.0 & 35.1 & 0.015 \\
\hline Diabetes mellitus $(\%)^{\mathrm{b}}$ & 1.9 & 3.0 & 3.0 & 4.1 & 3.0 & 0.56 \\
\hline Ratio of total to HDL cholesterol & $4.7 \pm 1.6$ & $4.7 \pm 1.6$ & $4.6 \pm 1.4$ & $4.6 \pm 1.5$ & $4.5 \pm 1.5$ & 0.51 \\
\hline History of cardiovascular disease (\%) & 4.9 & 9.6 & 4.6 & 8.7 & 7.4 & 0.027 \\
\hline \multicolumn{7}{|l|}{ 24-h ambulatory measurements } \\
\hline Systolic blood pressure (mmHg) & $125.0 \pm 12.4$ & $126.9 \pm 13.0$ & $128.3 \pm 13.4$ & $127.4 \pm 13.9$ & $125.5 \pm 15.5$ & 0.53 \\
\hline Diastolic blood pressure (mmHg) & $77.2 \pm 9.0$ & $75.7 \pm 8.2$ & $74.7 \pm 8.3$ & $73.4 \pm 9.1$ & $70.3 \pm 9.9$ & $<0.001$ \\
\hline Mean arterial pressure $(\mathrm{mmHg})$ & $93.1 \pm 9.1$ & $92.8 \pm 9.3$ & $92.6 \pm 9.4$ & $91.4 \pm 10.1$ & $88.7 \pm 11.2$ & $<0.001$ \\
\hline Pulse pressure (mmHg) & $47.9 \pm 7.4$ & $51.2 \pm 8.2$ & $53.6 \pm 9.0$ & $53.9 \pm 8.8$ & $55.1 \pm 9.4$ & $<0.001$ \\
\hline
\end{tabular}

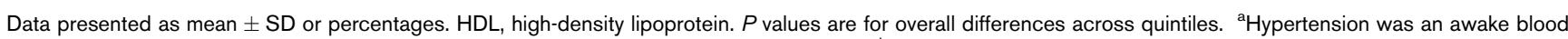

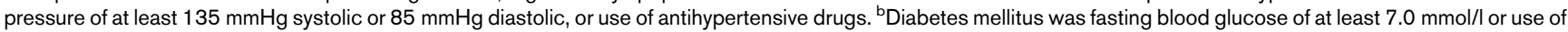
antidiabetic drugs.

diabetes mellitus, the ratio of total to HDL serum cholesterol, and systolic blood pressure. The diastolic blood pressure and mean arterial pressure decreased with increasing the AASI, whereas with a higher AASI the pulse pressure also increased. The AASI was higher $(P<0.001)$ in women than men $(0.58$ versus 0.54$)$, and in normotensive than hypertensive individuals $(0.57$ versus 0.54$)$. The AASI increased with age $(r=0.12, P<0.001)$, but decreased with body height $(r=-0.15, P<0.001)$.

\section{Incidence of endpoints}

During follow-up (median, 9.4 years; 5th-95th percentile interval, $3.1-10.1$ years), 17389 person-years were accrued. Of 219 deaths, 94 (42.9\%) were due to cardiovascular illnesses. The incidence of the composite cardiovascular outcome totaled 212 events, including 60 cardiovascular deaths, 128 coronary events, and 24 strokes. Coronary heart disease consisted of 37 fatal and 113 nonfatal events, including 27 fatal and 49 nonfatal cases of acute myocardial infarction. Of 40 strokes, 20 were nonfatal events.

\section{Analysis of outcome}

Using Cox regression models, we computed the hazard ratios associated with a $1 \mathrm{SD}$ increase in the AASI and pulse pressure: first without any adjustment; next with adjustment for sex, age, body mass index, mean arterial pressure, smoking, diabetes mellitus, the ratio of total to HDL serum cholesterol, and a history of cardiovascular disease; and, finally, we additionally adjusted the AASI for pulse pressure and vice versa (Table 2). Both before and after adjustment, the AASI predicted fatal and nonfatal stroke, even when the pulse pressure was forced into the model. In contrast, while adjusting for other risk factors, pulse pressure lost its predictive value for the three outcomes under investigation. This was mainly due to the influence of the mean arterial pressure.

In adjusted analyses of the AASI treated as a binary variable, elevated values significantly and independently predicted fatal and nonfatal stroke. Pulse pressure, when treated as a binary variable and adjusted for other covariates, lost its predictive value for the three outcomes.

Table 2 Relative hazard ratios relating various outcomes to the ambulatory arterial stiffness index (AASI) and 24-h pulse pressure

\begin{tabular}{|c|c|c|c|c|}
\hline $\begin{array}{l}\text { Endpoint } \\
\text { (number of events) }\end{array}$ & $\begin{array}{c}\text { AASI } \\
(1 \mathrm{SD}=0.14 \mathrm{U})\end{array}$ & $\begin{array}{l}\text { 24-h pulse pressure } \\
(1 \mathrm{SD}=9.0 \mathrm{mmHg})\end{array}$ & $\begin{array}{c}\text { Dichotomized AASI } \\
(0,1 ; n=1411,418)\end{array}$ & $\begin{array}{l}\text { Dichotomized 24-h pulse pressure } \\
\qquad(0,1 ; n=1413,416)\end{array}$ \\
\hline \multicolumn{5}{|c|}{ Cardiovascular events $(n=212)$} \\
\hline Unadjusted & $1.07(0.94-1.23)$ & $1.54(1.38-1.73)^{\dagger}$ & $0.64(0.45-0.92)^{*}$ & $2.26(1.71-2.98)^{\dagger}$ \\
\hline Adjusted $^{\mathrm{a}}$ & $1.06(0.92-1.22)$ & $1.04(0.89-1.20)$ & $0.92(0.64-1.33)$ & $1.12(0.81-1.54)$ \\
\hline Fully adjusted ${ }^{b}$ & $1.06(0.91-1.23)$ & $1.02(0.87-1.19)$ & $0.90(0.62-1.31)$ & $1.10(0.79-1.52)$ \\
\hline \multicolumn{5}{|l|}{ Stroke $(n=40)$} \\
\hline Unadjusted & $1.58(1.15-2.16)^{*}$ & $1.62(1.25-2.10)^{*}$ & $1.57(0.81-3.04)$ & $2.37(1.26-4.47)^{*}$ \\
\hline Adjusted $^{\mathrm{a}}$ & $1.57(1.12-2.19)^{*}$ & $1.00(0.70-1.41)$ & $2.44(1.24-4.78)^{*}$ & $1.00(0.48-2.06)$ \\
\hline Fully adjusted ${ }^{b}$ & $1.62(1.14-2.28)^{*}$ & $0.87(0.60-1.26)$ & $2.51(1.26-5.00)^{*}$ & $0.88(0.42-1.83)$ \\
\hline \multicolumn{5}{|c|}{ Coronary heart disease $(n=150)$} \\
\hline Unadjusted & $0.96(0.82-1.12)$ & $1.42(1.24-1.64)^{*}$ & $0.50(0.31-0.80)^{*}$ & $1.92(1.37-2.69)^{\dagger}$ \\
\hline Adjusted $^{a}$ & $0.94(0.80-1.12)$ & $0.94(0.78-1.13)$ & $0.70(0.43-1.13)$ & $0.93(0.63-1.38)$ \\
\hline Fully adjusted ${ }^{b}$ & $0.96(0.80-1.14)$ & $0.95(0.79-1.15)$ & $0.71(0.44-1.16)$ & $0.95(0.64-1.41)$ \\
\hline
\end{tabular}

Data presented as the relative hazard ratio (95\% confidence interval) associated with a 1 SD increase in the AASI or $24-\mathrm{h}$ pulse pressure or with an abnormally elevated AASI or 24-h pulse pressure dichotomized based on the upper boundary of the $95 \%$ prediction interval for individual data in relation to age in a normotensive reference population. Significance of the relative hazard ratios. $* P<0.01 .{ }^{\dagger} P<0.001$. ${ }^{a}$ Adjusted for sex, age, body mass index, mean arterial pressure, smoking, diabetes mellitus, ratio of total to high-density lipoprotein serum cholesterol, and a history of cardiovascular disease. ${ }^{\mathrm{b}} \mathrm{AASI}$ additionally adjusted for pulse pressure and vice versa. 
Figure 1 shows the absolute risk of fatal and nonfatal stroke at different levels of mean arterial pressure and pulse pressure, while controlling for sex and age.

Sensitivity analysis of the AASI as a predictor of stroke The relative hazards ratios relating stroke to the AASI tended to be slightly higher in women than men, and in normotensive compared with hypertensive individuals (Fig. 2), but the interaction terms between the AASI and sex and between the AASI and hypertension status were not significant in unadjusted $(P>0.13)$ as well as adjusted $(P>0.25)$ analyses. Furthermore, compared with the adjusted model in Table 2, the relative hazard ratio for stroke did not change when we excluded 129 individuals with previous cardiovascular disease (1.63; 95\% confidence interval, $1.10-2.40 ; P=0.015)$. When we used an alternative adjustment by applying the Framingham risk score [18] to our total study population, the AASI remained a significant predictor of stroke with a hazard ratio of 1.61 (95\% confidence interval, $1.15-2.25 ; P=0.005)$.

We also adjusted the hazard ratios relating stroke to the AASI in a stepwise fashion, first for traditional risk factors and then for various blood pressure components. The cardiovascular risk factors included sex, age, body mass index, smoking, diabetes mellitus, the ratio of total to HDL serum cholesterol, and a history of cardiovascular disease. Further adjustments for blood pressure were cumulative and additionally included either the 24-h systolic or diastolic blood pressure or both, or the 24-h pulse pressure (Fig. 3). The AASI remained a significant $(P \leq 0.016)$ predictor of stroke in all these models.

\section{Discussion}

The key finding of our study was that the AASI, a measure of the dynamic relation between diastolic and systolic blood pressure through the whole day, was a robust predictor of stroke in middle-aged and older individuals over and beyond traditional risk factors, even including mean arterial pressure and 24-h pulse pressure. For each 1 SD increment in the AASI, the risk of stroke increased by $61 \%$. Our findings extend the validation of the AASI as a predictor of stroke from hypertensive patients [7] to a random population sample, and from stroke mortality [7] to fatal and nonfatal strokes. Indeed, in the Dublin Outcome Study [7], which involved 11291 referred patients with a mean age of 54.6 years, the AASI was a stronger predictor of stroke mortality than pulse pressure, with an opposite trend for cardiac mortality. Moreover, in the Irish cohort [10] the AASI was more predictive of stroke in normotensive than hypertensive individuals [7]. We confirmed that the AASI was a significant predictor of stroke in normotensive individuals, but not hypertensive patients, albeit that in our current study the interaction term between the AASI and the hypertension status did not reach statistical significance. In line with several other studies [19-22], we noticed that pulse pressure did not predict the incidence of stroke. In a seminal article published in 1989, Darné et al. [19] investigated the prognostic value of the steady and pulsatile components of blood pressure in 9351 women and (a)

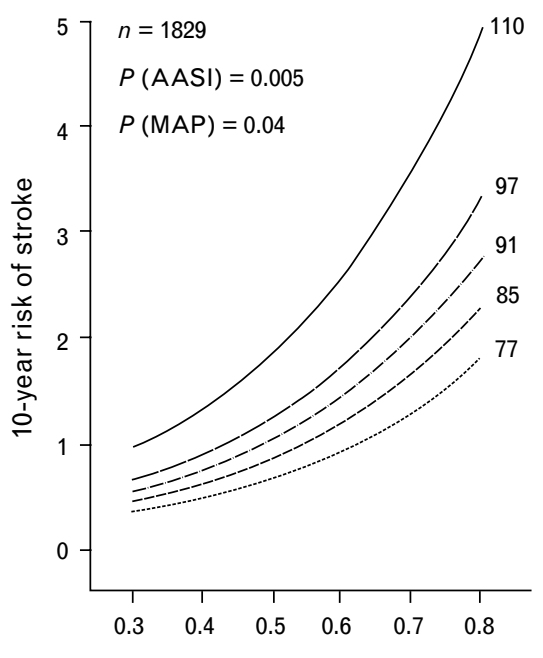

(b)

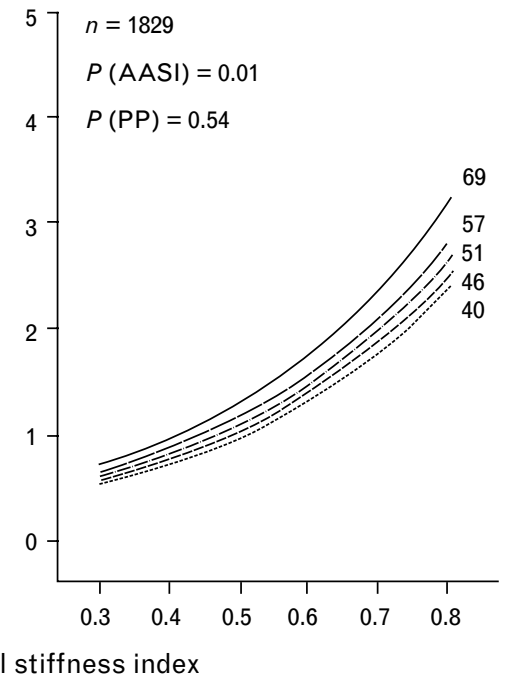

Absolute risk of stroke associated with the ambulatory arterial stiffness index (AASI) at different levels of (a) mean arterial pressure (MAP) or (b) 24-h pulse pressure (PP), while controlling for sex and age. Plotted values of the AASI span the 5th-95th percentile interval. The continuous risk functions of MAP and PP correspond with the 5th, 25th, 50th, 75th and 95th percentiles. Significance levels are given for the independent associations of stroke risk with the AASI (PAASI) and MAP (PMAP) or PP (PPP). 
Fig. 2

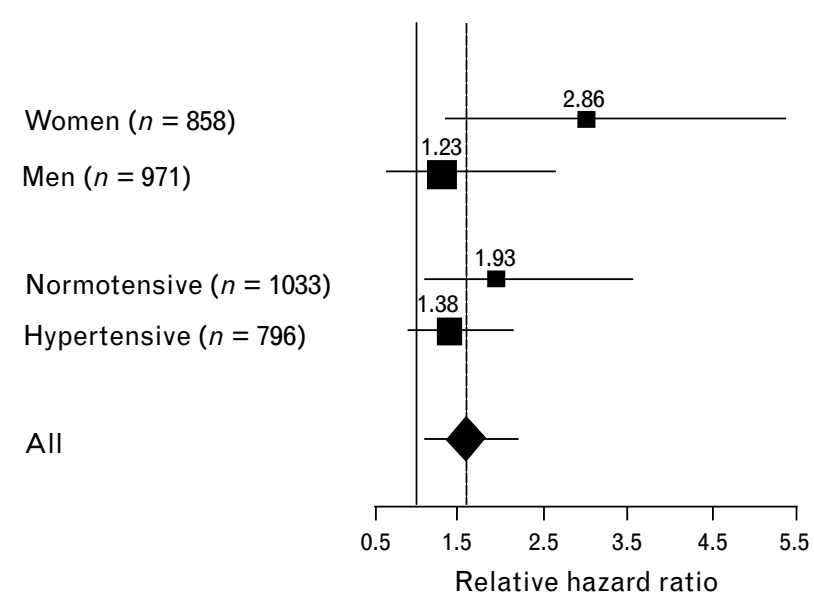

Hazard ratios for stroke associated with a 1 SD increase in the ambulatory arterial stiffness index by sex or hypertension status. Hypertension was an awake blood pressure of at least $135 \mathrm{mmHg}$ systolic or $85 \mathrm{mmHg}$ diastolic, or use of antihypertensive drugs. Estimates were adjusted for sex (only for normotension versus hypertension), age, body mass index, mean arterial pressure, smoking, diabetes mellitus, the ratio of total to high-density lipoprotein serum cholesterol, and history of cardiovascular disease. Horizontal lines denote $95 \%$ confidence intervals. The size of the squares is proportional to the number of events in each group.

18336 men, aged 40-49 years and followed-up for a mean period of 9.5 years. Based on principal component analysis, the steady component was a strong predictor of all types of cardiovascular death in both sexes. In women, the pulsatile component, independent of the steady component, predicted death from coronary heart disease, but was inversely correlated with stroke mortality [19]. Two other large-scale population studies [20,21] demonstrated that, after adjustment for the mean arterial pressure, the pulse pressure lost its predictive value in relation to stroke mortality [20] or fatal combined with nonfatal stroke [21], even in individuals older than 55 years [21] or 65 years [20]. Similarly, in Verdecchia et al.'s [23] study of 2311 patients with essential hypertension, adjustment for mean arterial pressure removed the significance of the association between the 24-h pulse pressure and the risk of cerebrovascular events, including stroke and transient ischemic attack.

The Framingham investigators [24] demonstrated that the prognostic significance of pulse pressure is age dependent. In subjects younger than 50 years of age, diastolic blood pressure was a strong predictor of coronary heart disease. Age 50-59 years was a transition period when systolic, diastolic and pulse pressures were similar predictors of cardiovascular risk, while from 60 years onwards the diastolic pressure was negatively related to the risk of coronary events, so that pulse pressure became a better predictor than systolic pressure [24]. In our study population, the conventionally measured pulse pressure based on office measurement, but not the 24-h ambulatory
Fig. 3

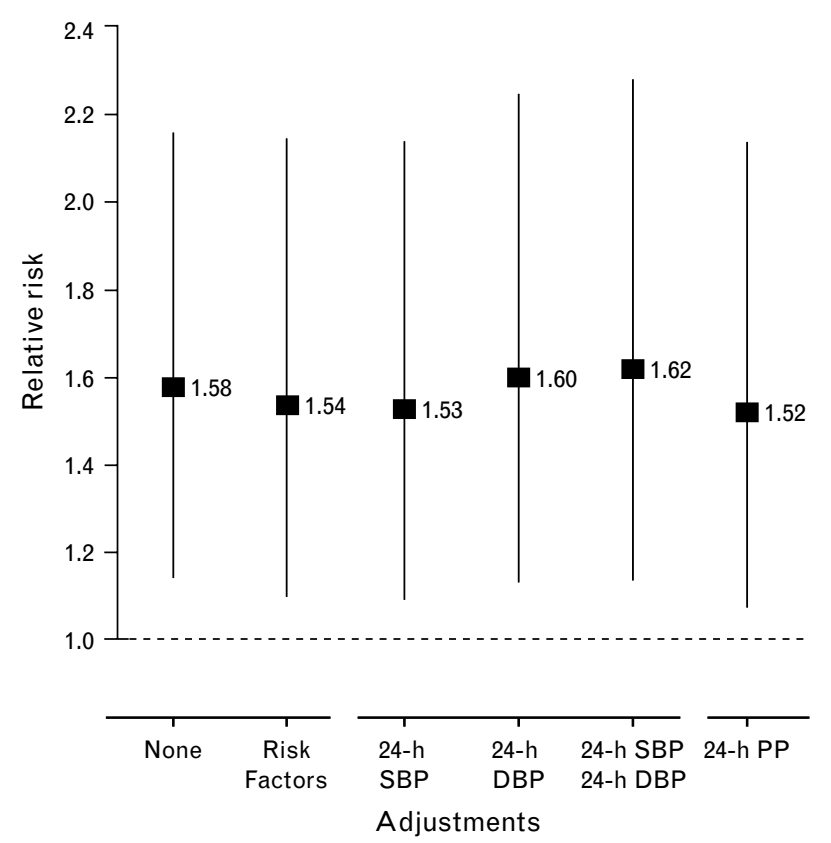

Hazard ratios for stroke associated with a 1 SD increase in the ambulatory arterial stiffness index. The hazard ratios were adjusted in a stepwise fashion for traditional risk factors and various blood pressure components. The risk factors included sex, age, body mass index, smoking, diabetes mellitus, the ratio of total to high-density lipoprotein serum cholesterol, and a history of cardiovascular disease. Further adjustments were cumulative and additionally included either the 24-h systolic blood pressure (SBP) or diastolic blood pressure (DBP) or both, or the 24-h pulse pressure (PP). Vertical lines denote 95\% confidence intervals.

pulse pressure, predicted the incidence of coronary events [2]. In the Dublin Outcome Study [7], the 24-h pulse pressure was a significant predictor of cardiac mortality with adjustments applied for mean arterial pressure and other risk factors. Thus, in the interpretation and comparison of studies on the prognostic value of pulse pressure, one should account for the distribution and range of age [24], the technique and conditions of blood pressure measurement and, above all, concomitant adjustment for other blood pressure components [19$21,25]$.

In contrast to AASI, the 24-h pulse pressure does not account for the diurnal variability in the relation between diastolic and systolic blood pressure [6,7]. The AASI depends on the combined effects of left ventricular ejection, the active and passive components of arterial stiffness, and the reflection of the arterial pulse wave. We previously demonstrated that in healthy volunteers the correlation coefficient between the AASI and the aortic pulse wave velocity was 0.51 [6]. Furthermore, in randomly recruited Chinese individuals, both before and after adjustment for arterial wave reflections by considering height and heart rate as covariates, the AASI correlated more closely with the central and peripheral systolic 
augmentation indexes than the 24-h pulse pressure did [6]. In line with the Chinese study [6], we noticed in the current study that the AASI was positively related to age and negatively to height. In our current population, however, the AASI was on average approximately $0.20 \mathrm{U}$ higher than in Chinese [6]. It decreased with higher mean arterial pressure, and was slightly higher in normotensive compared with hypertensive individuals. These findings might be attributable to the stratification of our sample by sex and age, the older age of our participants (55.5 versus 46.1 years [6]), the higher proportion of patients with hypertension (43.5 versus $32.8 \%$ [6]), and the greater prevalence of smoking (44.1 versus $26.7 \%$ [6]). Furthermore, patients with known hypertension or individuals of older age without occupational activity might have been more willing to undergo ambulatory blood pressure monitoring. Indeed, the 827 individuals excluded from the analysis, compared with those included, were younger ( 52.8 versus 55.5 years, $P<0.01$ ), more likely to be female $(56.2$ versus $46.8 \%, P<0.01$ ), and to have lower systolic/diastolic levels of office blood pressure (128.4/79.8 versus $131.6 / 81.2 \mathrm{mmHg}, P<0.01$ ). Furthermore, smoking acutely raises blood pressure [26] and increases arterial stiffness [27], and during the awake period might therefore have influenced the relation between diastolic and systolic blood pressure.

The derivation of the AASI and the 24-h pulse pressure rests on the measurement of systolic and diastolic blood pressure at the level of the brachial artery. Wave reflections originating from peripheral sites augment the systolic blood pressure and pulse pressure in the central arteries, depending on anthropometric characteristics, heart rate, arterial stiffness, and peripheral resistance $[6,14,28]$. In contrast, the diastolic blood pressure and mean arterial pressure are constant throughout the arterial tree [29]. These hemodynamic concepts might explain why in large outcome studies [30-32] the mean arterial pressure was a better predictor of stroke than pulse pressure, and why pulse pressure lost its significance in the prediction of stroke when corrected for mean arterial pressure [19-21,33]. We hypothesize that the AASI is a measure characteristic for each individual and that it overcomes some of the limitations of systolic blood pressure and pulse pressure, which when measured at the level of the brachial artery do not reliably reflect what happens in the central arteries. According to the opinion of some [34], albeit not all [35], experts, however, the AASI might not be a precise index of arterial stiffness, but rather a blood pressure component just predicting outcome better than, for instance, pulse pressure.

The present study must be interpreted within the context of its potential limitations and strengths. We assumed that recruitment of individuals aged 30, 40, 50 , or 60 years and followed-up for a median of approximately 10 years would be representative for a middle- aged to older population. In comparison with other studies [30-32], the number of participants and stroke events was substantially smaller. As in all longitudinal studies, the subjects remaining under investigation decreased over time, were to some extent self-selected, and in our study represented only $70.2 \%$ of those originally recruited. About 800 further individuals were either not willing to undergo ambulatory monitoring or had recordings of too low quality. On the other hand, follow-up of our participants in terms of fatal and nonfatal events was complete. Compared with conventional blood pressure measurement, ambulatory monitoring more precisely reflects an individual's usual blood pressure, excludes observer bias, and minimizes the white-coat effect [8].

In hypertensive patients as well as the general population, the AASI is a strong predictor of stroke over and beyond mean arterial pressure and other cardiovascular risk factors. Further studies should address the reproducibility of the AASI in repeated ambulatory blood pressure recordings and investigate whether the AASI can be pharmacologically influenced by drugs known to increase arterial distensibility [36] or to reduce the central pulse pressure [37,38]. A worldwide consortium of investigators [17] is currently constructing an international database of ambulatory blood pressure recordings in relation to cardiovascular outcomes and will further study the predictive value of the AASI in population-based cohorts of different ethnicity. In the meantime, manufacturers of devices for ambulatory blood pressure monitoring can include the computation of the AASI in their software packages. Clinicians might consider the AASI in the risk stratification of their patients under the proviso that further clinical and epidemiological validation of this novel prognostic index of arterial function is warranted.

\section{Acknowledgement}

The authors gratefully acknowledge the clerical assistance of Mrs Sandra Covens (Leuven, Belgium).

\section{References}

1 Sutton-Tyrrell K, Najjar SS, Boudreau RM, Venkitachalam L, Kupelian V, Simonsick EM, et al. Elevated aortic pulse wave velocity, a marker of arterial stiffness, predicts cardiovascular events in well-functioning older adults. Circulation 2005; 111:3384-3390.

2 Hansen TW, Staessen JA, Torp-Pedersen C, Rasmussen S, Thijs L, Ibsen $\mathrm{H}$, et al. Prognostic value of aortic pulse wave velocity as index of arterial stiffness in the general population. Circulation 2006; 113:664-670.

3 Laurent S, Boutouyrie P, Asmar R, Gautier I, Laloux B, Guize L, et al. Aortic stiffness is an independent predictor of all-cause and cardiovascular mortality in hypertensive patients. Hypertension 2001; 37:1236-1241.

4 Boutouyrie P, Tropeano Al, Asmar R, Gautier I, Benetos A, Lacolley P, et al. Aortic stiffness is an independent predictor of primary coronary events in hypertensive patients. A longitudinal study. Hypertension 2002; 39: $10-15$.

5 Laurent S, Katsahian S, Fassot C, Tropeano Al, Gautier I, Laloux B, et al. Aortic stiffness is an independent predictor of fatal stroke in essential hypertension. Stroke 2003; 34:1203-1206.

6 Li Y, Wang JG, Dolan E, Gao PJ, Guo HF, Nawrot T, et al. Ambulatory arterial stiffness index derived from 24-hour ambulatory blood pressure monitoring. Hypertension 2006; 47:359-364. 
7 Dolan E, Thijs L, Li Y, Atkins N, McCormack P, McClory S, et al. Ambulatory arterial stiffness index as a predictor of cardiovascular mortality in the Dublin Outcome Study. Hypertension 2006; 47:365-370.

8 O'Brien E, Asmar R, Beilin L, Imai Y, Mallion JM, Mancia G, et al. European Society of Hypertension recommendations for conventional, ambulatory and home blood pressure measurement. J Hypertens 2003; 21: 821-848.

9 Tunstall-Pedoe H, Kuulasmaa K, Mahonen M, Tolonen H, Ruokokoski E, Amouyel P. Contribution of trends in survival and coronary-event rates to changes in coronary heart disease mortality: 10-year results from $37 \mathrm{WHO}$ MONICA project populations. Monitoring trends and determinants in cardiovascular disease. Lancet 1999; 353:1547-1557.

10 41st World Medical Assembly. Declaration of Helsinki: recommendations guiding physicians in biomedical research involving human subjects. Bull Pan Am Health Org 1990; 24:606-609.

11 Rasmussen SL, Torp-Pedersen C, Borch-Johnsen K, Ibsen H. Normal values for ambulatory blood pressure and differences between casual blood pressure and ambulatory blood pressure: results from a Danish population survey. J Hypertens 1998; 16:1415-1424.

12 Hansen TW, Jeppesen J, Rasmussen S, Ibsen H, Torp-Pedersen C. Ambulatory blood pressure and mortality: a population study. Hypertension $2005 ;$ 45:499-504.

13 Imai Y, Sasaki S, Minami N, Munakakata M, Hashimoto J, Sakuma H, et al. The accuracy and preformance of the A\&D TM 2421, a new ambulatory blood pressure monitoring devise based on the cuff-oscillometric method and the Korotkov sound technique. Am J Hypertens 1992; 5:719-726.

14 Nichols WW, O'Rourke M. Contours of pressure and flow waves in arteries. In McDonalds blood flow in arteries. Theoretical, experimental and clinical principles. 5th ed. London: Hodder Arnold; 2005. pp. 165-191.

15 Report of the Expert Committee on the Diagnosis and Classification of Diabetes Mellitus. Diabet Care 1997; 20:1183-1197.

16 Madsen M, Davidsen M, Rasmussen S, Abildstrom SZ, Osler M. The validity of the diagnosis of acute myocardial infarction in routine statistics: a comparison of mortality and hospital discharge data with the Danish MONICA registry. J Clin Epidemiol 2003; 56:124-130.

17 Staessen JA, O'Brien ET, Amery AK, Atkins N, Baumgart P, De Cort P, et al. Ambulatory blood pressure in normotensive and hypertensive subjects: results from an international database. J Hypertens 1994; 12 (Suppl 7): $\mathrm{S} 1-\mathrm{S} 12$.

18 Wilson PW, D'Agostino RB, Levy D, Belanger AM, Silbershatz $\mathrm{H}$, Kannel WB. Prediction of coronary heart disease using risk factor categories. Circulation 1998; 97:1837-1847.

19 Darné B, Girerd X, Safar M, Cambien F, Guize L. Pulsatile versus steady component of blood pressure: a cross-sectional analysis and a prospective analysis on cardiovascular mortality. Hypertension 1989; 13:392-400.

20 Benetos A, Safar M, Rudnichi A, Smulyan H, Richard JL, Ducimetiere P, et al. Pulse pressure: a predictor of long-term cardiovascular mortality in a French male population. Hypertension 1997; 30:1410-1415.

21 Miura K, Soyama Y, Morikawa Y, Nishijo M, Nakanishi N, Naruse $\mathrm{Y}$, et al. Comparison of four blood pressure indexes for the prediction of stroke risk in middle-aged and older Asians. Hypertension 2004; 44:715-720.

22 Verdecchia P, Schillaci G, Reboldi G, Franklin SS, Porcellati C. Different prognostic impact of 24-hour mean blood pressure and pulse pressure on stroke and coronary artery disease in essential hypertension. Circulation 2001; 103:2579-2584.

23 Verdecchia P, Schillaci G, Reboldi G, Franklin SS, Porcellati C. Different prognostic impact of 24-hour mean blood pressure and pulse pressure on stroke and coronary artery disease in essential hypertension. Circulation 2001; 103:2579-2584.

24 Franklin SS, Larson MG, Khan SA, Wong ND, Leip EP, Kannel WB, et al. Does the relation of blood pressure to coronary heart disease change with aging? The Framingham Heart Study. Circulation 2001; 103:12451249.

25 Verdecchia P, Schillaci G, Reboldi G, Franklin SS, Porcellati C. Different prognostic impact of 24-hour mean blood pressure and pulse pressure on stroke and coronary artery disease in essential hypertension. Circulation $2001 ; 103: 2579-2584$.

26 Wilhelmsen L. Smoking and blood pressure. Blood Press 1996; 5:69-70.

27 Mahmud A, Feely J. Effect of smoking on arterial stiffness and pulse pressure amplification. Hypertension 2003; 41:183-187.

28 Mitchell GF, Lacourcière Y, Arnold JMO, Dunlap ME, Conlin PR, Izzo JL Jr. Changes in aortic stiffness and augmentation index after acute converting enzyme or vasopeptidase inhibition. Hypertension 2005; 46:1111 1117.

29 Van Bortel LM, Balkestein EJ, van der Heijden-Spek JJ, Vanmolkot FH, Staessen JA, Kragten JA, et al. Non-invasive assessment of local arterial pulse pressure: comparison of applanation tonometry and echo-tracking J Hypertens 2001; 19:1037-1044.
30 Prospective Studies Collaboration. Age-specific relevance of usual blood pressure to vascular mortality: a meta-analysis of individual data for one million adults in 61 prospective studies. Lancet 2002; 360:1903-1913.

31 Asia Pacific Cohort Studies Collaboration. Blood pressure indices and cardiovascular disease in the Asia pacific region. A pooled analysis. Hypertension 2003; 42:69-75.

32 Zhang XF, Attia J, D'Este C, Yu XH. Prevalence and magnitude of classical risk factors for stroke in a cohort of 5092 Chinese steelworkers over 13.5 years of follow-up. Stroke 2004; 35:1052-1056.

33 Verdecchia P, Schillaci G, Reboldi G, Franklin SS, Porcellati C. Different prognostic impact of 24-hour mean blood pressure and pulse pressure on stroke and coronary artery disease in essential hypertension. Circulation $2001 ; 103: 2579-2584$.

34 Laurent S. Surrogate measures of arterial stiffness. Do they have additive predictive value or are they only surrogates of a surogates. Hypertension 2006; 47:325-326.

35 Benetos A, Lacolley P. From 24-hour blood pressure measurments to arterial stiffness. A valid short cut? Hypertension 2006; 47:327-328.

36 Van Bortel LMAB, Struijker-Boudier HAJ, Safar ME. Pulse pressure, arterial stiffness, and drug treatment of hypertension. Hypertension 2001; 38:914-921.

37 London GM, Asmar RG, O'Rourke MF, Safar, on behalf of the REASON project investigators. Mechanism(s) of selective systolic blood pressure reduction after a low-dose combination of perindopril/indapamide in hypertensive subjects: comparison with atenolol. J Am Coll Cardio/ 2004; 7:92-99.

38 Williams B, Lacy PS, Thom SM, Cruickshank K, Stanton A, Collier D, et al. Differential impact of blood pressure-lowering drugs on central aortic pressure and clinical outcomes. Principal results of the Conduit Artery Function Evaluation (CAFE). Circulation 2006; 113:1213-1225. 